\section{New injectable restorative claims to be strong as a rock}

GC's new G-aenial Universal Injectable composite range is an easy-to-use, composite restorative material that is as strong as a rock.

Available in 16 shades in three opacities, G-aenial Universal Injectable composite restorative material offers exceptional strength and wear resistance thanks to its combination of ultra-fine Barium fillers and GC's Full-coverage Silane Coating (FSC) technology. Suitable for any cavity class without size limitation, it is strong enough to restore the tooth on its own.

G-aenial Universal Injectable also has a unique thixotropic viscosity optimised for free-hand build-up of cusps.

The new syringe design of G-aenial Universal Injectable enables easy extrusion without the mess and inconvenience of uncontrolled flow-out. Whilst the materials viscosity is optimised for easy separation of the material from the tip, the tips themselves feature a long bendable needle for unparalleled access to difficult to reach posterior

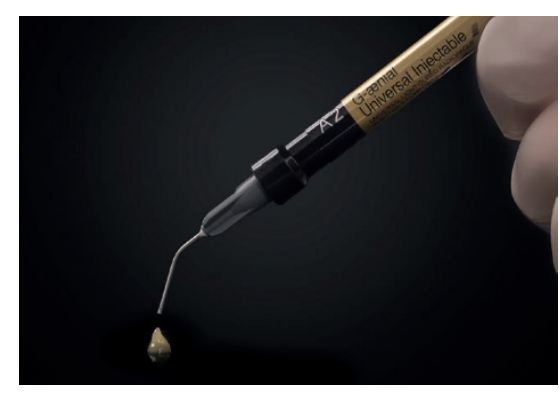

cavities.

Call GC UK Ltd on 01908218999 or e-mail info@gcukltd.co.uk or visit www.gceurope.com.

\title{
System to encourage child tooth hygiene
}

The new Zooby Disclosing Tablets support professional teeth cleaning for children and increase their personal responsibility by colouring new plaque red and older plaque blue, highlighting areas that are often missed when brushing the teeth.

Zooby - a Young Innovations brand, leading developer, manufacturer and distributor of high-quality dental products - knows well how preventative therapy can avoid tooth decay and secondary diseases.

The patients receive individual instructions and tips for their personal dental care from the practitioner and afterwards, all tooth surfaces and interproximal areas are thoroughly cleaned and polished.

The fruit-flavoured tablets are available in packs of 250 and are free of gluten and erythrosine (E127). They can also be used at

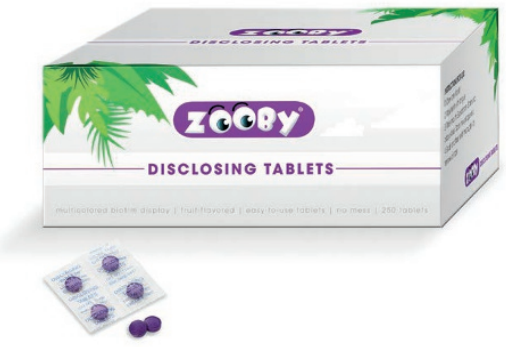

home to improve the daily care routine.

Professional tooth cleaning is carried out in a risk-oriented manner because the breakthrough of the second teeth brings about changes such as incomplete enamel formation, unfavourable topographical position of the chewing surfaces or retention niches and thus increasing the risk of plaque deposits. Also orthodontic braces as well as the increase in sugar intake and acidic foods effect plaque build-up.

\section{Midlands boost for dental access}

NHS England in the North Midlands has recently awarded $£ 2.25$ million to local NHS dental practices in an attempt to increase NHS dental access for around 30,000 patients across Staffordshire and Shropshire.

The funding will benefit 39 NHS dental practices across all 11 local authority areas, with the intention to boost NHS dental access for patients and in particular, encourage a higher take-up of dental check-ups for children aged up to two.

The activity is part of a wider national initiative designed to increase access for this age group by promoting earlier prevention.

NHS England said given that dental disease was now the most common reason for hospital admissions for children aged five to nine years old, the NHS was working hard to address this locally and nationally.

Rebecca Woods, Head of Primary Care for NHS England in Staffordshire and Shropshire said: 'This investment in dental services is really positive news for patients of Staffordshire and Shropshire and we are working closely with our Local Dental Network which includes dental providers across the local NHS, local authority, Public Health England's Consultant in Dental Public Health and NHS England commissioners to improve local dental services and promote earlier prevention at local level.'

Sara Hurley, Chief Dental Officer for England, said: 'We are currently seeing a
Zooby said its child-friendly prophylaxis system offers a wide range of tools to significantly increase patient compliance in children. The polishing cups are made of proprietary special rubber that adapts to the tooth contour accurately.

The polishing pastes are available in six popular flavours such as chocolate, melon and berry as well as three different grains each with $1.23 \%$ fluoride.

They remove stains, are remarkably efficient and are also vegan-, sugar-, lactose- and gluten-free. They are delivered as a practical hygienic single dose, and the matching finger holder is included free. In order to reduce spatter, the pastes have a particularly adhesive consistency, and the hygienic disposable packaging minimises the risk of cross-contamination. cavity crisis in pre-school children which is leading to entirely avoidable tooth extractions. Regular visits to your dentist from a very early age is key to developing habits that lead to a lifetime of good oral health. We are providing additional support to dentists to help them see more infants so that these painful and distressing interventions can be reduced.

'Latest data show over 140 children per day are having decayed teeth removed. These statistics are now being tackled head on with a newly launched awareness programme supporting dentists across England to see more children from a young age and infants by age one.' 